\title{
METODOLOGÍAS DE ESTIMACIÓN DE LA LÍNEA DE POBREZA ABSOLUTA EN COLOMBIA: UNA REFLEXIÓN'
}

\author{
Julián Augusto Casas Herrera ${ }^{2}$
}

Universidad Pedagógica y Tecnológica de Colombia, UPTC - Artículo Tipo 2.

Reflexión - Recibido: 16 Junio de 2015 - Aceptado: 21 Junio de 2015

doi: http://dx.doi.org/10.17981/econcuc.36.1.2015.28

\section{RESUMEN}

Este artículo surgió como respuesta esclarecedora a las polémicas cifras publicadas en torno de la pobreza monetaria en Colombia en 2011, según las cuales desaparecieron tres millones de pobres. Se indagó la raíz que sustenta las cifras: la metodología del cálculo de la Línea de Pobreza Absoluta. Ahondando sobre los elementos metodológicos, se encontró que la metodología empleada por la Comisión Económica Para América Latina y el Caribe (CEPAL) se convirtió en referente para la estimación de la pobreza en los países de América Latina. Por ello, este documento explicita las principales diferencias existentes entre las cuatro metodologías oficiales implementadas en Colombia sobre la medición de la pobreza. El texto muestra que las cifras de pobreza sirven como instrumento de focalización para quienes son identificados como pobres; sin embargo, se evidencia que cambios metodológicos pueden incidir en la correcta identificación del número de pobres. Esto hizo la última metodología implementada en el país, lo cual permite concluir que los encargados de llevar a cabo la medición deben asumir un compromiso ético que le dé coherencia a sus estimaciones con lo que se percibe en la sociedad.

\section{Palabras Clave:}

Metodología, Indigencia, Pobreza, Colombia.

JEL: B49, D13, D63

\section{Si va a referenciar este artículo}

Casas, J. (2015). Metodologías de estimación de la línea de pobreza absoluta en Colombia: Una reflexión. Económicas CUC, 36(1), 217-244.

doi: http://dx.doi.org/10.17981/econcuc.36.1.2015.28

\footnotetext{
${ }^{1}$ Artículo de reflexión producto de la investigación titulada "Propuesta alternativa para las líneas de indigencia y de pobreza existentes en Colombia (2002-2011)", bajo la línea de investigación desigualdad económica y política social, presentado a la Universidad Nacional de Colombia.

${ }^{2}$ Economista y especialista en finanzas de la Universidad Pedagógica y Tecnológica de Colombia (UPTC). Magister en Ciencias Económicas de la Universidad Nacional de Colombia. Docente de la escuela de economía de la UPTC. Correo: julian.casas01@uptc.edu.co.
} 


\section{INTRODUCCIÓN}

La motivación de este estudio surgió cuando la Misión de Expertos para el Empalme de las Series de Empleo y Pobreza (MESEP) y el Departamento Nacional de Planeación (DNP) publicaron en 2011 las cifras sobre pobreza. Estas están basadas en el cálculo de las Líneas de Indigencia (LI) y Líneas de pobreza (LP). La primera calcula una canasta de alimentos, por persona y por mes, cuyo valor fue de $\$ 78.000$. La segunda resulta de multiplicar el valor de la LI por el Coeficiente de Orshansky (CO), que tuvo un valor de 2.4. Sin embargo, los resultados generaron descontento nacional provocando un debate que surgió a raíz del uso del valor del $\mathrm{CO}$, puesto que la $\mathrm{ME}$ SEP usó el CO promedio de los países de América Latina (2.4), en vez del CO colombiano (2.87), que haría que la LP pasara de $\$ 187.200$ a $\$ 226.200$ y, en consecuencia, habría alrededor de 19 en vez de 16 millones de pobres. La desaparición de tres millones de pobres justifica el desarrollo de este trabajo, como elemento clarificador del debate académico y social generado en el país. (Muñoz, 2011)

Para llevar a cabo este ejercicio clarificador el documento tiene como objetivo explicitar las principales diferencias existentes entre las cuatro metodologías oficiales implementadas en Colombia sobre la medición de la pobreza monetaria, puesto que de estas se derivaron políticas conducentes a reducir los niveles de indigencia y de pobreza en el país. La importancia de esto reside en que pequeños cambios metodológicos, producen cambios en la correcta identificación del número de pobres. El documento tiene cuatro partes, contando la introducción como la primera. En la segunda se muestran los principales elementos de la metodología de la Comisión Económica para América Latina y el Caribe (CEPAL) que originó en América Latina (AL) la estimación de la pobreza. En la tercera se exponen las diferencias entre las cuatro metodologías implementadas en Colombia. Por último, se trazan unas conclusiones que recogen los principales elementos del documento.

\section{METODOLOGÍA CEPAL}

La metodología de la CEPAL se transformó en un referente para los países de $\mathrm{AL}$ a la hora de evaluar cuantitativamente la pobreza (Altimir, 1979). El enfoque adoptado por la CEPAL (1991), para estimar la magnitud de la pobreza en AL, corresponde al enfoque biológico ${ }^{3}$ que considera requerimientos de supervivencia para definir una LP así como una LI, ya que como dice Sen (1992), el hambre es el aspecto más evidente de la pobreza. Las estimaciones se basan en el método del ingreso o costo de las necesidades básicas que compara el ingreso de los hogares respecto del costo de satisfacción de sus necesidades básicas.

La estimación de la pobreza monetaria requiere información de ingresos o gastos -léase renta o consumo ${ }^{4}$-, que generalmente proviene de las Encuestas de Ingresos y Gastos (EIG) que realizan los países cada 10 o 12 años. El encargado de efectuar el ejercicio de medición debe evaluar la consistencia de los ingresos y los gastos ${ }^{5}$, ya que la información procede de una encuesta, es decir una muestra de la población del país,

\footnotetext{
${ }^{3}$ Este surgió a raíz del trabajo de Rowntree (1901).

${ }^{4}$ Coudouel, Hentschel y Wodon (2002) señalan que si la información de consumo de las encuestas de ingresos y gastos (EIG) está detallada, este sería mejor que la renta para medir la pobreza, por tres razones: i) el consumo está más ligado al bienestar del individuo; ii) se puede medir mejor que la renta; y iii) refleja mejor el nivel de vida real de los hogares, ya que revela la capacidad de estos para cubrir sus necesidades básicas. No obstante, el uso de la renta permite diferenciar entre las fuentes de ingresos haciendo fácil la comparación con datos procedentes de otras fuentes, lo que permitiría verificar la calidad de los datos de la EIG. Lo ideal es calcular las LP y LI con ambos para evaluar la consistencia de los resultados.

${ }^{5}$ Se evalúa observando el grado de subestimación de cada uno. Se opta por el que esté menos subestimado.
} 
que por sí conlleva un margen de error que afecta la calidad de las estimaciones.

El enfoque de la LP ha sido el más utilizado para hacer evaluaciones cuantitativas sobre la pobreza en el mundo. El enfoque clasifica a un hogar como pobre si su ingreso o gasto es menor que el valor de una LP dada Instituto Nacional de Estadística (INE). La LP representa el valor agregado de todos los bienes y servicios considerados indispensables para satisfacer las necesidades básicas del hogar. Hay tres enfoques de la LP: absoluto, relativo y subjetivo (Casas $\&$ Barichello, 2015). El primero es el que se analiza en este documento. La estimación de la LP se compone de dos líneas: Línea de Pobreza alimentaria (LPA) y Línea de Pobreza No Alimentaria (LPNA).

El cálculo de la LPA selecciona los alimentos que conformarán una Canasta Normativa de Alimentos (CNA) que debe satisfacer requerimientos nutricionales; mientras que para la LPNA no hay un criterio objetivo sobre un valor de referencia mínimo. Para seleccionar los contenidos de la canasta hay dos opciones: normativa y semi-normativa. La primera pretende que las personas coman saludablemente. La segunda respeta los hábitos de consumo, aunque no sean saludables. Dado que esta última es la más usada se describen los tres pasos principales para su construcción.

El primer paso consiste en determinar la tasa de metabolismo basal, es decir, el requisito energético o calórico ${ }^{6}$ mínimo necesario para mantener vivo a un individuo en reposo durante un día. Esta tasa se deduce de la información de la estructura sociodemográfica de la población urbana y rural de cada país, y estima concretamente requerimientos mínimos de calorías, proteínas y nutrientes para una persona de edad y sexo promedio a nivel

${ }^{6}$ Se suele medir en kilocalorías requeridas diariamente por un individuo. nacional y por día?. Una vez se calcula el promedio ponderado de los requerimientos calóricos por persona, se pasa a determinar los requerimientos totales para un hogar. Los requisitos surgen de recomendaciones de organismos como la Food and Agriculture Organization (FAO), Organización Mundial de la Salud (OMS), y la Universidad de Naciones Unidas (UNU) ${ }^{8}$.

El segundo paso hace una selección de un grupo poblacional objetivo, más conocido como Población de Referencia $(\mathrm{PR})^{9}$, con el propósito de definir la composición de la CNA y calcular el Coeficiente de Engel (CE) o el inverso del $\mathrm{CE}$, es decir el $\mathrm{CO}^{10}$. Para elegir esta población se organizan los hogares, de menor a mayor, de acuerdo al valor gastado en los bienes. Además, la selección de la misma debe cumplir dos condiciones:

i. el gasto en alimentación debe satisfacer los requerimientos nutricionales, respetar los hábitos alimentarios, y superar ligeramente a los requerimientos medios nutricionales mínimos estimados; y

ii. debe ser capaz de asumir otros gastos necesarios para satisfacer las necesidades más básicas no alimentarias (salud, vestido, educación, vivienda, transporte).

\footnotetext{
${ }^{7}$ Los requerimientos subestiman las necesidades de energía y proteínas de los hogares en que la edad de sus miembros es mayor al promedio nacional, y viceversa.

${ }^{8}$ Las recomendaciones de la FAO y la OMS tienen en cuenta la información censal de la estructura sociodemográfica de la población urbana y rural de los países. Las primeras recomendaciones sobre necesidades de energía provinieron de la FAO en 1950. De ahí en adelante ha realizado actualizaciones de los requerimientos proteínicos y energéticos para los años 1958, 1966, 1971, 1981, 1985 y 2001.

${ }^{9} \mathrm{El}$ criterio de la CEPAL requiere que los hogares incluidos en la misma, por un lado, satisfagan sus necesidades alimentarias, y por otro, que los hábitos de consumo en otros bienes y servicios no resulten de decisiones sujetas a un marco de extrema escasez ni de gran abundancia económica.

${ }^{10} \mathrm{El} \mathrm{CE}$ sale de dividir el gasto de alimentos entre los gastos totales. Mientras que el $\mathrm{CO}$ se obtiene de dividir los gastos totales entre el gasto de alimentos. La estimación del CE o del CO sale de la PR.
} 
En el tercer paso se establece el contenido y costo de la canasta de alimentos. El costo se puede determinar de dos modos: i) diseñar una canasta y asignarle un precio, ii) calcular el precio de la canasta sin especificar su contenido. El primer modo, el más común, determina los alimentos que compondrán la CNA analizando para cada alimento tres aspectos basados en los patrones de consumo de los hogares: i) frecuencia de adquisición junto con el porcentaje de hogares que lo adquirió; ii) gasto mensual por persona en dicho bien, es decir el porcentaje del gasto que representa en el total del gasto en alimentación; y iii) cantidades físicas adquiridas expresadas por la equivalencia gramos-día por persona.

De lo anterior, se desprenden los criterios de inclusión de alimentos ${ }^{11}$ que sirven para determinar la presencia o no de cada alimento dentro de la CNA. Básicamente, lo que se hace es tomar a cada uno de los alimentos registrados en la EIG y se chequea si el alimento, al menos satisface uno de los criterios y de ser así, el alimento entra en la canasta. Una vez dentro de la canasta se determina el grupo alimentario ${ }^{12}$ al que pertenece el alimento.

La CEPAL (1990) se aseguró que los alimentos de la CNA tuvieran al menos 10\% de calorías proteicas y entre $15 \%$ y $25 \%$ de calorías grasas; que los cereales y legumbres no aportaran más de $60 \%$ del total de

\footnotetext{
11 La CEPAL (1991) trabaja con cinco criterios de inclusión, pero cada país es autónomo de tomar tantos criterios como considere relevante. Los criterios son: i) si el alimento es consumido por el $30 \%$ o más de los hogares; ii) si el alimento representa por lo menos el $1 \%$ del total de gastos en alimentos; iii) si el alimento aporta por lo menos el 1\% del total de calorías o proteínas consumidas; iv) si el alimento representa el $0.5 \%$ o más del peso total de los alimentos adquiridos por los hogares; y v) si el gasto del alimento representa, al menos $5 \%$ o más dentro del grupo al que pertenece el alimento.

${ }^{12}$ La CEPAL (1991) clasifica 10 grupos alimentarios: harinas y cereales; lácteos; carnes y huevos; frutas; hortalizas; leguminosas; grasas; tubérculos, raíces y plátanos; azúcares; y otros. En estos últimos se reúnen aquellos alimentos de menor consumo.
}

las calorías; y para que la calidad de las proteínas fuera adecuada, por lo menos $35 \%$ de las mismas, tendrían que ser de origen animal. Igualmente, las CNA de cada país fueron evaluadas en términos del contenido de siete micronutrientes: calcio, hierro, retinol (léase vitamina A), tiamina (vitamina B1), riboflavina (vitamina B2), niacina (vitamina B3), y ácido ascórbico (vitamina C).

En seguida se revisa si el contenido de la CNA supera los requerimientos calóricos señalados por la norma calórica, suministrada por la FAO, y de ser así se pasa a hacer ajustes calóricos ${ }^{13}$, proteicos y dietéticos a cada uno de los alimentos que componen la CNA. De este modo, se estipulan las cantidades de calorías y nutrientes necesarios de cada alimento; obteniéndose las CNA de cada país ${ }^{14}$. Cabe indicar que la CNA no representa una dieta ideal, sino que busca: i) reflejar los hábitos de consumo de los hogares de la PR, ii) evitar enfermedades asociadas a inadecuada nutrición, y iii) obtener una CNA a un menor costo. Sin embargo, es pertinente precisar que si se han de hacer ajustes a la CNA, estos deben privilegiar los hábitos de la población más que la norma nutricional y el costo.

La valoración de la CNA se hace utilizando los precios implícitos de la propia EIG para la PR, que salen de dividir el gasto del alimento entre la cantidad adquirida del mismo. Cabe explicar dos aspectos. Por un lado, si el objetivo es obtener una canasta más barata se reemplazan alimentos costosos por otros más baratos del mismo grupo alimentario. Por el otro, la LPA sue-

\footnotetext{
13 Suponga que la FAO establece que las calorías que deben ser consumidas por día son de 2200, pero el contenido de la CNA arroja 2300. Entonces se pasa a dividir 2200 entre 2300, lo cual da un factor de ajuste de 0.95 que tendrá que multiplicarse a cada alimento para que la CNA quede ajustada a la norma calórica (2200). Los ajustes se hacen con el fin de que no se presenten problemas de sobrepeso, desnutrición, entre otros.

${ }_{14}$ De acuerdo a la información existente en el país se obtienen tanto CNA y CE para los dominios urbano y rural.
} 
le representar el costo por persona que esta necesita para cumplir con el requerimiento calórico promedio de la población. Aquí se debe señalar que en el enfoque de la LP la unidad de análisis es el hogar, pero no el individuo. Esto es una limitación debido a que es imposible evaluar la pobreza a nivel individual.

La alternativa para solucionar esta limitación pasa por asociar la LP del hogar a través de la LP per cápita. Sin embargo, al hacer esto se asume que el costo monetario para satisfacer las necesidades de un individuo es homogéneo para todos los miembros del hogar y que no hay economías de escala en el consumo. Además, el enfoque de la LP requiere de escalas de equivalencia para agregar los resultados a nivel nacional. La escala de equivalencia más utilizada es el $\mathrm{CE}^{15}$, que es esencial para estimar la LPNA. De hecho, para construir las LP y LI se requiere que la información de las EIG cumpla la ley de Engel ${ }^{16}$, y que los montos estimados de los diferentes rubros de gasto se encuentren dentro de niveles razonables al compararlos con otras fuentes de información ${ }^{17}$ (encuestas similares). Antes de concluir esta sección se deben precisar dos aspectos más: necesidades no alimentarias, junto con la corrección y ajuste de los ingresos.

Como ya se dijo, no hay un criterio objetivo para determinar un valor de referencia mínimo para la LPNA o necesidades no alimentarias. No obstante, el método

\footnotetext{
15 Este supone que la división entre los gastos en alimentación y los gastos totales de dos hogares es la misma. Así este índice expresa que el costo de manutención del primer hogar en comparación con el segundo es igual. Ver Grupo de Río (2007).

${ }^{16}$ Indica que a medida que la renta va aumentando, los gastos que se dedican a los artículos de primera necesidad son decrecientes, mientras que los que se dedican a bienes de lujo son crecientes.

${ }^{17}$ Esto es lo que se denomina consistencia externa de la EIG. Este punto fue el polémico en la última metodología implementada en Colombia. Vea Metodología 2011 más adelante.
}

más utilizado para establecer la LPNA se basa en el CE observado de la PR. Este se basa en el trabajo de Mollie Orshansky quien estableció las LP de los Estados Unidos; por ello a veces se denomina $\mathrm{CO}^{18}$. Nótese que la LP contempla alimentos, a través de la LPA, y además es consciente que los hogares son más que alimentos. Esta consciencia adquiere forma con el valor del CE que pretende contemplar los rubros de vivienda, transporte y vestuario ${ }^{19}$. Así si se divide el valor de la LPA entre el CE se obtendrá el valor definitivo de la LP; o también se puede obtener si se multiplica la LPA por el $\mathrm{CO}^{20}$.

Por otro lado, determinadas las LI y LP y dados los problemas que tienen las $\mathrm{EIG}^{21}$ la CEPAL decidió evaluar la magnitud de los sesgos implícitos en los montos de ingreso declarados para utilizar adecuadamente los datos de ingresos y, en consecuencia,

${ }^{18}$ Este tiene diversas implicaciones. Por un lado, según el INE (sin fecha) supone "que los hogares que logran cubrir adecuadamente sus necesidades de alimentación satisfacen, al mismo tiempo, los estándares mínimos de las otras necesidades básicas, algo que no es necesariamente apoyado por la evidencia empírica (...) [Por el otro, indica que] puede existir una inconsistencia en esta manera de llegar a una línea de pobreza. Los requerimientos mínimos de alimentación se derivan en forma normativa, calculando cuánto costarían los requerimientos mínimos; mientras los ítems no alimentarios se determinan observando en realidad cuánto gasta la gente. Para eliminar la inconsistencia, tendríamos que asumir que lo que la gente realmente llega a gastar es lo que necesita gastar en los ítem no alimentarios, un supuesto claramente irreal".

${ }^{19}$ Dentro de la canasta no alimentaria se incluye una porción del ingreso para el vestuario que busca cobijar un ropero esencial; también se incluye una porción para vivienda de dos y tres dormitorios, que trata de cobijar una parte para el arriendo de una familia de referencia, junto con el pago de servicios públicos y algunas comodidades (nevera, cocina, lavadora); y se incluye un componente para el transporte de los miembros de la familia de referencia para ir al trabajo, al colegio, de compras, entre otras (INE, sin fecha).

${ }^{20}$ Actualmente, la CEPAL trabaja con CO urbanos de un valor de 2 , y para la zona rural de 1.75 .

${ }^{21}$ Tales como calidad y exactitud de las mediciones de ingreso, cobertura geográfica, representatividad de la muestra, la falta de respuesta a las preguntas de ingresos, los sesgos y errores asociados al muestreo. 
pasó a realizar una corrección y ajuste de los ingresos. Para ello acudió al Ajuste a Cuentas Nacionales (ACN) que compara las partidas de ingreso de la EIG con las resultantes de una estimación de la cuenta de ingresos y gastos de los hogares del sistema de cuentas nacionales; así solo si los primeros están por encima de los últimos se generaban coeficientes de ajuste de acuerdo con la discrepancia para cada fuente de ingreso, y así se suavizaban los sesgos por sub-declaración o por la falta de respuesta a preguntas sobre los ingresos por parte de asalariados, trabajadores independientes y jubilados.

En síntesis, el primer y tercer paso sirven para definir el valor monetario de la LI, mientras que el segundo paso sirve para dar el CO por el cual se multiplica para dar la LP. Así la gente que no alcance un ingreso igual o superior al valor de la LI, se considerará indigente o pobre extremo. En cambio, los que se encuentran por encima de la LI y por debajo del valor de la LP se consideran pobres.

Finalmente, cabe precisar tres cosas. La primera es que las EIG se realizan cada 10 a 12 años, dado que su costo es muy alto. Por ello, los valores de las LI y LP se actualizaban, en los años que no hay encuesta, de acuerdo con la variación acumulada del Indice de Precios al Consumidor (IPC) de cada país. La CEPAL (2010) señala que hasta diciembre de 2006 aplicó la misma variación a ambas líneas, pero desde 2007 la LI se actualiza con la variación del IPC para alimentos, y la LP con la variación del IPC de cada año. La segunda es que desde el año 2005 la CEPAL (2010) comenzó a revisar la metodología que ha venido utilizando desde los ochenta, por cuatro razones:

i. Las LI y LP se derivan de patrones de consumo de las EIG de esos años. Por ello, se debe construir nuevas CNA que se ciñan a los hábitos de las EIG más recientes; ii. Se requiere introducir cambios metodológicos que reflejen los avances en la medición de la pobreza en el mundo (técnicas de recolección, entre otros);

iii. Es necesario ampliar el concepto de ingreso utilizado de las EIG ${ }^{22}$; y

iv. Realizar la revisión del ACN, ya que actualmente no se considera esencial hacerlo.

Y la tercera se debe a los propósitos que se persiguen con la estimación de las LP y LI, los cuales buscan: i) cuantificar la magnitud de la pobreza, ii) identificar a los hogares en dicha situación, y iii) brindar información para el diseño de políticas públicas dirigidas a reducir tanto la indigencia como la pobreza $^{23}$. De modo que la información que arroja la clasificación del número de pobres e indigentes sirve como instrumento de focalización para que los gobiernos, a través de programas de políticas públicas, aminoren los efectos negativos que generan la pobreza.

\footnotetext{
${ }^{22}$ Este concepto se ha discutido en el informe del grupo de Canberra (2001).

${ }^{23}$ En Colombia se pueden clasificar en 9 grandes grupos: i) Transferencias Monetarias Condicionadas, entre estos están: Familias en Acción y Programa de Protección Social al Adulto Mayor; ii) Cuidado y Desarrollo Infantil: Programa de Atención Integral a la Primera Infancia y Programa Hogares Comunitarios; iii) Alimentación y Nutrición Infantil: Programa de Alimentación Escolar, Programa Desayunos Infantiles con Amor; iv) Educación: Programa Crédito Access y Programa Gratuidad; v) Salud: Régimen Subsidiado de Salud; vi) Empleo: Programa Jóvenes en Acción, Programa Jóvenes Rurales y Programa Subsidio al Desempleo; vii) Inclusión Económica y Social: Programa Oportunidades Rurales, Programa Subsidio Integral de Tierras, Programa Vivienda de Interés Social, Programa Vivienda Rural, Red para la Superación de la Pobreza Extrema (Unidos), viii) Adulto Mayor: Programa Nacional de Alimentación para el Adulto Mayor "Juan Luis Londoño de la Cuesta"; ix) Población Pobre: Red de Seguridad Alimentaria (Ver: http://estadisticas.comunidadandina. org/estadisticasandinas/CANPS/default.aspx). Además, los Conpes relacionados con la pobreza son los números 102, 111, 135, 150 y 217. El lector puede visitar el siguiente link para ver los Conpes aprobados: https:// sisconpes.dnp.gov.co/DocumentosConpesAprobados/ IraDocumentosConpesAprobados/tabid/166/Default.aspx
} 


\section{METODOLOGÍAS EMPLEADAS EN COLOMBIA}

Este acápite explica los aspectos que se tuvieron en cuenta en las cuatro metodologías implementadas en Colombia para el enfoque de la LP absoluta, para 1988, 1998, 2005 y 2011. El documento toma como referente los trabajos de Muñoz \& Rivas (2006) y el de la MESEP (2011). El primer trabajo sirve de referente para las metodologías de 1988 (M88), 1998 (M98) y 2005 (M05). El segundo expone la metodología de 2011 (M11).

El trabajo de Muñoz \& Rivas (2006), muestra una comparación, a partir de sus valores y características, entre las canastas obtenidas con las M88 y M98. La necesidad comparativa surgió porque para 1998 se estimaron las LP y LI con la EIG 1994-1995 (EIG94-95). Además, esta estimación se realizó mediante una metodología diferente a la utilizada en 1988 que trabajó con la EIG 1984-1985 (EIG84-85); lo anterior originó como resultado que las CNA de 1998 fueran más costosas que las de 1988. Las estimaciones de 1998 de las LI y LP no fueron publicadas; pero el Departamento Administrativo Nacional de Estadística (DANE) actualizó anualmente sus valores, encontrándose que para 2004 un $10 \%$ más de pobres con la M98, en comparación con la M88.

La controversia tuvo un importante eco y se decidió indagar sobre las diferencias entre la M88 y la M98, dando como resultado la M05. El ejercicio comparativo construyó de nuevo CNA, pero aplicando la M88 con los datos de la EIG94-95. Para hacer comprensible las diferencias entre las cuatro metodologías señaladas se pasa a describir sus principales aspectos, resaltando que las diferencias residen en cuatro aspectos: i) PR; ii) criterio para la inclusión de alimentos a la CNA; iii) determinación de las cantidades necesarias de los alimentos que componen las CNA; y iv) cálculo de la LP.

\section{Metodología 1988}

La crítica principal que generó la M88 tuvo que ver con la elección de la PR, ya que la construcción de la CNA escogió los hábitos de consumo de los hogares más pobres y, por ende, el método se basó en una preselección de pobres, lo cual metodológicamente requiere que los hogares incluidos no se enfrenten a un marco de extrema escasez ni de abundancia económica. De hecho, la M88 tomó como $P R$ entre los percentiles 1 al 25. Antes de pasar a señalar el criterio para la inclusión de alimentos a la CNA, Muñoz (1991), manifiesta que una CNA debe tener cuatro características: i) satisfacer los requerimientos nutricionales mínimos; ii) respetar, en lo posible, los hábitos alimenticios de la PR; iii) tener en cuenta, en lo posible, la disponibilidad de alimentos; y iv) tener un mínimo costo. En ese sentido, la M88 tuvo en cuenta cuatro criterios para la inclusión de alimentos. Así, un alimento entró en la CNA si al menos satisfacía uno de los siguientes criterios:

i. lo consumía el 30\% o más de los hogares,

ii. representaba por lo menos el $1 \%$ del total de gastos en alimentos,

iii. aportaba por lo menos el 1\% del total de calorías o proteínas consumidas, y

iv. representaba el $0.5 \%$ o más del peso total de los alimentos adquiridos por los hogares.

Para la determinación de las cantidades necesarias de los alimentos que compondrían las CNA se tuvo en cuenta los requerimientos nutricionales de la población colombiana, que se rigieron por un lado, por los lineamientos de la FAO/OMS/UNU (1985), con información que proporcionó el Instituto Colombiano de Bienestar Familiar, específicamente sobre talla y peso. Además, de las calorías y proteínas la M88 
tuvo en cuenta siete nutrientes: calcio, hierro, retinol, tiamina, riboflavina, niacina, vitamina $\mathrm{C}^{24}$.

Cabe indicar que la M88 decidió trabajar con información del gasto corriente del hogar, en vez del ingreso, porque según Muñoz (1991) "la información de gasto es más confiable que la de ingresos, ya que esta última presenta un mayor grado de subestimación. Este hecho se refleja en que la proporción de pobres es menor, para doce de las trece ciudades, cuando se toma el gasto como referencia, con (...) excepción de Medellín”.

Con el criterio de inclusión y determinadas las cantidades de alimentos, se valoró la CNA o LI. Además, se introdujeron tres restricciones nutricionales con el fin de que existiera un balance entre calorías y proteínas, que quedó establecido de la siguiente manera: i) las proteínas de origen animal debían estar entre $20 \%$ y $45 \%$, ii) las calorías provenientes de grasas debían estar entre $20 \%$ y $30 \%$, las de proteínas entre $8 \%$ y $12 \%$, y las de carbohidratos entre $55 \%$ y $65 \%$; y iii) el azúcar y la panela no debían aportar más del $20 \%$ de la energía obtenida de todas las fuentes de carbohidratos. (Muñoz, 1991)

Con estas restricciones se clasificaron los alimentos que compondrían las CNA en nueve grupos de alimentos: lácteos (leche y queso); carnes y huevos; leguminosas; cereales; tubérculos, raíces y plátanos; hortalizas; frutos; grasas; y azúcares. Finalmente, en cuanto al cálculo de la LP se estimó dividiendo la LI entre el CE. Con estos criterios se estimaron las LI y LP para trece ciudades, que fueron actualizándose anualmente con el IPC.

\footnotetext{
${ }^{24}$ Los requerimientos promedio de calorías y nutrientes per cápita de la población colombiana de calorías, proteínas y micro-nutrientes fueron: calorías (2209), proteínas $(62 \mathrm{mg})$, calcio $(874.6 \mathrm{mg})$, hierro $(16.4 \mathrm{mg})$, niacina $(15.1 \mathrm{mg})$, retinol $(820 \mathrm{mg})$, riboflavina $(1.36 \mathrm{mg})$, tiamina $(1.2 \mathrm{mg})$, vitamina $\mathrm{C}(56.1 \mathrm{mg})$.
}

\section{Metodología de 1998}

Las CNA se construyeron, con la EIG94-95, teniendo en cuenta la crítica que generó la M88. Por eso, la M98 cambió la $P R$ y añadió un criterio para la inclusión de alimentos en las CNA. Respecto del primer cambio, tomó los hábitos de consumo promedio entre los percentiles 1 al 90 de la población. Respecto del segundo cambio, según Muñoz \& Rivas (2006), la poca diversidad de alimentos de la M88 llevó a introducir un nuevo criterio de selección. El nuevo criterio incluyó un alimento en la CNA si el gasto de este representaba, al menos $5 \%$ o más dentro del grupo al que pertenece el alimento.

La determinación de las cantidades necesarias de los alimentos que compondrian las CNA de la M98 cambió respecto a la M88 ya que las recomendaciones promedio de calorías, proteínas y micro-nutrientes quedaron así: calorías (2297), proteínas (62), calcio (620), hierro (16), niacina (16.1), retinol (775), riboflavina (1.4), tiamina (1.2), vitamina C (53). Nótese que en la M98, se redujeron los requerimientos nutricionales de calcio en $29 \%$, hierro $2.4 \%$, retinol $5.5 \%$, y vitamina C 5.5\%; en cambio, en calorías, niacina y riboflavina aumentaron en $4 \%, 6.6 \%$ y $2.9 \%$ respectivamente. En proteínas y tiamina no hubo cambios. Debido a estos cambios las CNA de la M98 fueron más costosas que las de la M88.

Por último, para la estimación de la LP se modificó el cálculo del $\mathrm{CE}$, que quedó reflejado en la siguiente ecuación de Engel para alimentos: en la que representa la participación del gasto en alimentos sobre el gasto total (es decir, el CE) y es el gasto corriente total del hogar. Así si se sustituye en la ecuación el valor de la LI, se encuentra el gasto total y, por ende, w; así se obtuvo la LP. 


\section{Metodología de 2005}

La M05 volvió a tomar como $P R$ al $25 \%$ de los más pobres; el criterio para la inclusión de alimentos a la CNA fue el mismo usado en la M88; para la determinación de las cantidades necesarias de los alimentos que compondrían las CNA se tuvo en cuenta los requerimientos nutricionales de la población colombiana, guiados por los lineamientos de la FAO/OMS/UNU (1985). Es indispensable señalar que la M05 se desarrolló a partir de dos fuentes de información: i) para la LP urbana usó la EIG94-95 y ii) para la LP rural utilizó la información del primer seguimiento del programa familias en acción del 2003. El cálculo de la LP se hizo del mismo modo que en la M88.

Además, la M05 estimó valores de LI y LP para 13 ciudades $^{25}$, el resto (10 ciudades más ${ }^{26}$ y para la zona rural. En cuanto a la LP de la zona rural, se debe destacar que para construir la LI, Muñoz y Rivas (2006) advierten:

"No se sabe realmente cómo se obtuvo la línea de pobreza rural utilizada hasta ahora; solo se tiene el dato de la fuente de información, que fue la Encuesta de Alimentación y Nutrición de 1981, pero no se conoce la metodología ni documento alguno sobre ella. [Infortunadamente], la información de gasto o consumo de alimentos es muy escasa para la zona rural, por tanto, es difícil tener información para construir las canastas normativas".

Por ello, indican que una de las ventajas de la M05 reside en que gracias a la evaluación del impacto del programa familias

\footnotetext{
${ }^{25}$ Bogotá, Medellín, Cali, Barranquilla, Bucaramanga, Manizales, Pasto, Cartagena, Cúcuta, Pereira, Montería, Neiva y Villavicencio.

${ }_{26}$ Tunja, Florencia, Popayán, Valledupar, Quibdó, Riohacha, Santa Marta, Armenia, Sincelejo e Ibagué.
}

en acción se efectuaron, en 2002 y 2003, encuestas a las familias rurales más pobres de los estratos 1 y 2 del Sistema de Potenciales Beneficiarios para Programas Sociales - SISBÉN. La de 2002 se denominó línea de base (LB) y la de 2003 primer seguimiento (PS). Este hecho permitió construir la CNA rural, porque la información de las encuesta de PS, según Muñoz \& Rivas (2006), poseía información sobre la adquisición de alimentos por parte de los hogares.

Dos aspectos más se deben señalar sobre la encuesta de PS. Por un lado, analizó dos tipos de municipios: control y tratamiento. Pero se prefirió trabajar con los municipios tratamiento porque los hogares de estos tuvieron factores de expansión ${ }^{27}$. Por el otro, para valorar la cantidad obtenida de alimentos no comprados ${ }^{28}$ se usó el precio implícito promedio de estos del municipio a que correspondía el hogar. Además, la elaboración de la CNA rural tuvo dos fases: i) tomó al total de la $\mathrm{PR}$ de los municipios tratamiento; y ii) para la estimación de precios y cantidades, específicamente, con las que son compradas se usaron los precios implícitos promedio de cada alimento.

El criterio para la inclusión de alimentos a la CNA rural manejó dos formas de selección: la M88 y la metodología CEPAL. La principal discrepancia entre ambas está en que la segunda, los alimentos que no se incluyen directamente en la CNA se incluyen en el rubro llamado otros. Es decir, mientras que las M88, M98 y M05 agrupan los alimentos de la CNA en nueve grupos, la metodología CEPAL los agrupa en diez.

La determinación de las cantidades necesarias de los alimentos que compondrian las CNA se hizo de la misma forma que en

\footnotetext{
${ }^{27}$ Un factor de expansión es la cantidad de personas en la población, que representa una persona en la muestra.

${ }^{28}$ Es decir, aquellos que son regalados, auto-consumo o auto suministro.
} 
la M88. No obstante, hubo una modificación que tuvo en cuenta un promedio basado en la estructura por edad y sexo de la población rural. Esta información salió del censo poblacional de 1993. Muñoz \& Rivas (2006) comentan que al valorar la CNA con cada una de las metodologías encuentran que con la M05 el valor de la LI, para el mes de septiembre de 2003, fue de \$2113.1 diarios per cápita; en cambio, con la de la CEPAL el valor fue de $\$ 2237.7$.

Al realizar la comparación entre ambas metodologías se encontró que la LI de la CEPAL se ajustó mejor en la gran mayoría de nutrientes -menos en proteínas-, razón por la cual esa canasta se prefirió a pesar de que su costo superara a la CNA de la M05 en $\$ 124.6$ diarios. Por último, para pasar de la LI a la LP la M05 siguió exactamente a la M88, pero téngase en cuenta que la PR para la zona rural fue el total de la encuesta PS.

Dos aclaraciones finales son necesarias: por un lado, el CE en la M05 para todas las ciudades fue más alto frente a la M98, debido a la PR que se utilizó. Por otro lado, las M88, M98 y M05 usaron el ACN, que contrasta los montos de ingreso por fuente de ingresos (salarios, ganancias y otros) de las EIG, respecto a los montos de las mismas fuentes de las cuentas nacionales del país. Así, al contrastar se obtenían factores de ajuste que se aplicaban a los ingresos de las EIG con el fin de ajustar o corregir el problema de sub-declaración que se registra en la EIG.

\section{Metodología $2011^{29}$}

Según los diseñadores de la M11 era necesario modificar la LP por seis razones: i) implementar métodos estadísticos sofis-

\footnotetext{
${ }^{29}$ Lo expuesto en esta sección se basa en el documento intitulado "Pobreza monetaria en Colombia: Nueva metodología y cifras 2002-2010”, presentado por la MESEP (2011).
}

ticados para imputar los datos faltantes; ii) eliminar el ACN; iii) incluir adelantos metodológicos recientes, para obtener una medición más precisa de la pobreza en el país; iv) actualizar los hábitos de consumo, ya que se contaba una nueva EIG, a saber la encuesta nacional de ingresos y gastos 2006-2007 (ENIG06-07) ${ }^{30}$; v) incorporar las recomendaciones recientes de la FAO; y vi) lograr comparabilidad con otros países latinoamericanos.

La M11, a diferencia de la M05, construyó las LP urbana y rural con la misma fuente de información (ENIG06-07), que según la MESEP garantizó una mejor comparabilidad entre los datos urbanos y rurales. La M11 evaluó la consistencia interna y externa de la ENIG06-07, a través de la estimación de curvas de Engel para la primera; y para la segunda, comparó las distribuciones de diferentes rubros de gasto frente a los obtenidos en otras encuestas ${ }^{31}$. Los miembros de la MESEP determinaron que la ENIG06-07 tiene consistencia interna, pero externa no. De hecho, resaltan que en la ENIG06-07 existe la subestimación de algunos rubros de gasto, en comparación con otras fuentes que capturan información similar, hasta del $50 \%{ }^{32}$. Esta conclusión fue determinante para optar por un CO exógeno para la zona urbana,

\footnotetext{
${ }^{30}$ Nótese que se pasó de la EIG a la ENIG dado que el DANE, para 2006-2007, integró la encuesta de hogares con la EIG. No obstante, según Núñez, citado por Fedesarrollo (2011), expresa que esta integración trajo varios problemas tales como que la ENIG se hizo sobre una muestra del $25 \%$ de los hogares de la encuesta de hogares, la muestra de la encuesta de hogares se cambió sensiblemente y los ingresos tuvieron variaciones inexplicables a lo largo de la distribución del ingreso, y los datos reportados de ingresos tuvieron que desecharse para 2006 y 2007 , lo cual provocó que no se dieran estimaciones de pobreza para esos años.

${ }^{31}$ Algunas de ellas son: la EIG1994-1995, la encuesta de calidad de vida 2003, entre otras.

${ }^{32}$ Los miembros de la MESEP señalan que, por ejemplo, la subvaloración del rubro de alimentos se manifiesta al comparar el gasto promedio en alimentos de la ENIG06-07 con la Encuesta de Calidad de Vida ECV08 la diferencia es significativa, $\$ 287.000$ para la primera y $\$ 654.000$ para la segunda.
} 
y mantener la proporción del CO rural ${ }^{33}$. En ese orden de ideas, la M11 siguió seis pasos para construir las LP y LI:

i. determinación de la lista de los gastos corrientes per cápita por unidad de gasto;

ii. construcción de un deflactor espacial de precios, para establecer la relación entre los dominios urbano y rural y, al aplicarse, se pudo tener una sola PR;

iii. ordenación de todos los hogares por el gasto corriente per cápita deflactado para determinar una $\mathrm{PR}$, tomando el $45 \%$ de la incidencia, que quedó ubicada entre los percentiles 30 a 59;

iv. construcción de las CNA, que se ajustaron a los requerimientos calóricos ${ }^{34}$ junto con tres criterios para la inclusión de alimentos;

v. valoración de las CNA o LI con la mediana de los precios implícitos; y

vi. multiplicación del CO exógeno por la LI para obtener la LP.

Con estos aspectos se actualizaron mensualmente las LP y LI por medio de la inflación anual. Cabe señalar que con la ampliación de los niveles de desagregación geográfica, implementados por el DANE con la encuesta integrada de hogares a partir de 2006 y debido a la disponibilidad de información del IPC, se pudo pasar de 13 a 24 ciudades. Dicho en otras palabras, la M11 actualizó las LP y LI para 26 domi-

\footnotetext{
${ }^{33} \mathrm{El}$ CO urbano promedio fue de 2.4, aunque el CO que arrojó la ENIG06-07 fue de 2.87, mientras que el CO rural fue de 2.09. Lo que se hizo en la M11 fue mantener la proporción y así condicionó el CO rural al valor de 1.74, que salió de hacer la siguiente regla de tres: COrural = $\left(\frac{2.09 * 2.4}{2.87}\right)$

${ }^{34}$ El contenido calórico de la canasta básica se obtuvo de multiplicar las cantidades consumidas por el aporte calórico de cada alimento, que tuvo como fuente las tablas del Instituto Colombiano de Bienestar Familiar 2000, 2005, FAO y DANE.
}

nios, divididos en dos grupos: 25 dominios urbanos y 1 en la zona rural ${ }^{35}$.

La M11 tomó como $P R$ los percentiles 30 a 59 de la distribución de gasto per cápita. Para la selección de esta se aplicó el método iterativo de Ravallion (1998), que supone un porcentaje de la incidencia de la pobreza inicial para el total nacional alrededor del cual estaría centrada dicha población, que se definió en el $45 \%$. Este proceso verifica la validez de la PR, a partir de la comprobación de la incidencia de la pobreza. Así, si el nuevo porcentaje de pobres está dentro del intervalo definido para la PR a priori (percentiles 30 a 59), el proceso termina y se asevera que se tienen las LI y LP. Si cae fuera del intervalo se repite el proceso eligiendo una PR centrada en el nuevo porcentaje de incidencia encontrado con las nuevas líneas.

Para la inclusión de alimentos a la CNA, la M11 tomó de la ENIG06-07 los hábitos de consumo de los dominios urbano y rural de la PR con el fin de construir las CNA de cada dominio. La M11 incluyó los alimentos que cumplieran con al menos uno de los siguientes tres criterios:

i. lo consumía el 30\% o más de los hogares;

ii. representaba por lo menos el $1 \%$ del total de gastos en alimentos; $y$

iii. aportaba por lo menos el 1\% del total de calorías consumidas.

Para la determinación de las cantidades necesarias de los alimentos que compondrían las CNA se tuvieron en cuenta los requerimientos nutricionales de la población colombiana, basados en las recomendaciones de la FAO/OMS/UNU de 2001. En la M11 se ajustaron las CNA para alcanzar reque-

\footnotetext{
${ }^{35}$ Las 13 áreas tradicionales son: Medellín, Barranquilla, Bogotá, Cartagena, Manizales, Montería, Villavicencio, Pasto, Cúcuta, Pereira, Bucaramanga, Ibagué y Cali. Las nuevas ciudades son: Tunja, Florencia, Popayán, Valledupar, Quibdó, Neiva, Riohacha, Santa Marta, Armenia y Sincelejo. Por último, los otros dominios son otras cabeceras, rural y nacional.
} 
rimiento calórico. Esto se logra usando las cantidades observadas en la PR y comparándolas con la norma calórica que aconseja la FAO para cada dominio. En Colombia, los requerimientos calóricos promedio se obtuvieron a partir de la estructura poblacional por edad y sexo de la ENIG06-07. La norma calórica para el dominio urbano determinó 2090 calorías diarias, y para el rural se situó en 2049. Para cumplir con la norma se aplicó un factor de ajuste de 1.969 a las cantidades del dominio urbano y de 0.9242 para el dominio rural. Además, nótese que las CNA de la M11 no tuvieron en cuenta los siete micronutrientes ni los otros requerimientos de proteínas. Es decir, solo se ajustaron por calorías.

La LP se obtuvo a partir de la multiplicación entre la LI y el CO; sin embargo, se debe aclarar que el CO se puede estimar endógena o exógenamente. Si se acude a la primera forma, este saldría de la información de la EIG; si se acude a la segunda forma, se utiliza información secundaria. La M11 optó por esta última opción, tomando datos calculados por la CEPAL para los países de AL, porque a juicio de la MESEP la ENIG06-07 presentó problemas de consistencia externa, evidenciado en la subestimación del gasto total en alimentos, que es determinante para la estimación del CO.

Los miembros de la MESEP decidieron utilizar un CO exógeno de $2.4^{36}$ a nivel urbano, y de 1.74 a nivel rural. Así, la LP urbana para 2007 se obtuvo de multiplicar la LI (\$73.984) y el CO (2.4), que dio como resultado $\$ 177.562$; y la LP rural del producto entre la LI (\$60.968) y el CO (1.74), dando $\$ 106.084$. Estos valores son pesos de mar-

\footnotetext{
${ }^{36}$ Los países que se utilizaron para hacer el promedio del CO para el área urbana fueron: Argentina (2.6), Bolivia (2.2), Brasil (3.5), Chile (3), Costa Rica (2.8), Ecuador (2.2), Guatemala (2.5), Honduras (2.2), México (2.8), Nicaragua (1.9), Panamá (2.8), Paraguay (2.4), Perú (2.2), República Dominicana (2.3), Uruguay (3.4). De hacer este promedio no da 2.4, sino 2.59. Sin embargo, al aplicar el criterio de la CEPAL denominado pauta depurada, se sacó el dato de Brasil, dando como resultado 2.35, pero se ajustó hacia arriba, quedando 2.4.
}

zo de 2007. Por ello, los valores de las LP y LI se calcularon para los años anteriores (2002-2005) y posteriores (2008-en adelante) a la ENIG06-07. Finalmente, para actualizar los valores mensuales de la LI la M11 usó el IPC de alimentos, mientras que para la LP utilizó el IPC de ingresos bajos.

Finalmente, cabe señalar que los ejercicios metodológicos expuestos se derivan en valores diarios de la CNA (la cual está integrada por varios alimentos) o LI junto con un valor del CE o el CO. Los resultados obtenidos por cada una de las metodologías implementadas en el país se resumen en la tabla 1.

Del análisis anterior se concluye que las estimaciones de las LI y LP son susceptibles de variaciones según la metodología empleada. De hecho, el debate en el país se generó a raíz de algunos elementos metodológicos de la M11. Quizá el más cuestionable fue el uso de un CO exógeno (2.4), pues tal como señala Núñez, citado por Fedesarrollo (2011): no tomar el [CO] que proviene de la encuesta, de alguna manera, es aceptar que los datos no son confiables en su conjunto. Por ende, al no utilizar el CO endógeno (2.87) se subestimó la pobreza para los diferentes dominios del país y, por ello, el número de personas que deberían ser incluidas en programas de política pública orientados a mejorar su bienestar se redujo. Entonces, nótese que cambios metodológicos incidieron en la identificación correcta del número de pobres en el país. El solo cambio del CO generó que para 2010 se desaparecieran alrededor de 3 millones de pobres.

Así mismo, llama la atención porqué la MESEP no tuvo en cuenta el CO de Brasil (3.5), ya que según la MESEP era muy alto. Si se hubiera tenido en cuenta se estaría hablando de un CO urbano de 2.59, que hubiera significado mayores porcentajes de pobreza. Así que mientras las cifras revelan que la pobreza en el país ha venido reduciéndose la percepción de la realidad dice otra cosa. Por ello, los encargados de reali- 
Tabla 1.

LI y LP per cápita, por día y mes, de las M88, M98, M05 y M11

\begin{tabular}{cccccc}
\hline Metodología & Dominio & LI & CO & LP = LI * CO & LP mensual \\
\hline \multirow{2}{*}{ M88 } & 13 ciudades & $\$ 908.37^{*}$ & 2.29 & $\$ 2080.16$ & $\$ 62405$ \\
\hline \multirow{2}{*}{ M05 } & 13 ciudades & $\$ 1195.04^{*}$ & 2.64 & $\$ 3161.48$ & $\$ 94844$ \\
\cline { 2 - 5 } & 10 ciudades & $\$ 1199.36^{* *}$ & 2.66 & $\$ 3198.29$ & $\$ 95949$ \\
\cline { 2 - 5 } & 13 ciudades & $\$ 1005.38^{*}$ & 2.35 & $\$ 2371.18$ & $\$ 71135$ \\
\hline \multirow{2}{*}{ M11 } & 10 ciudades & $\$ 1004.24^{* *}$ & 2.27 & $\$ 2282.36$ & $\$ 68471$ \\
\cline { 2 - 5 } & Urbano & $\$ 2466.13^{* * *}$ & 2.4 & $\$ 5918.72$ & $\$ 177562$ \\
\hline \multirow{*}{*}{$*$} & Rural & $\$ 2032.27^{* * *}$ & 1.74 & $\$ 3536.14$ & $\$ 106084$ \\
$* * * *$ & $\begin{array}{l}\text { El valor refleja el promedio de las LI de las trece ciudades a pesos de agosto de 1994. } \\
\text { El valor refleja el promedio de las LI de las diez ciudades a pesos de agosto de 1994. }\end{array}$ & \\
& Los valores corresponden a los estimados por la MESEP. & &
\end{tabular}

Fuente: Construcción propia con datos de Muñoz \& Rivas (2006), Muñoz (1991) y MESEP (2011)

zar la medición de la pobreza deben asumir un compromiso ético que le dé coherencia a sus estimaciones con lo que se percibe en la sociedad, puesto que lo que está en juego es la humanidad y dignidad de los más vulnerables de una sociedad ya que los datos oficiales de la pobreza sirven como instrumento de política pública para aminorar los efectos negativos que genera en la existencia de los pobres, a través de los subsidios que otorga a los que son identificados como indigentes o pobres.

\section{CONCLUSIONES}

Este documento tuvo como motivación al debate generado por las cifras publicadas en 2011 sobre pobreza obtenidas por la MESEP. Debate que se magnificó debido a la presunta desaparición de alrededor de tres millones de pobres. Esto justificó indagar el porqué de dicha desaparición, encontrándose que el enfoque utilizado por Colombia, para hacer evaluaciones cuantitativas de pobreza, es la LP absoluta. Enfoque que se sustentó en la metodología de la CEPAL. También se mostraron los principales elementos metodológicos que tuvieron en cuenta los cuatro ejercicios que han adelantado mediciones de pobreza en Colombia, verificándose diferencias entre las mismas.
De este modo, se evidenció que las estimaciones son susceptibles de variaciones metodológicas. Particularmente hay, por lo menos, un elemento metodológico de la M11 que es cuestionable (el uso del $\mathrm{CO}$ exógeno), que incidió en los resultados presentados por la MESEP y, por ende, en las políticas económicas que de ellos se derivaron. Así al no utilizar el CO endógeno los niveles de pobreza fueron menores para los diferentes dominios del país y, por ello, el número de personas que debieron ser incluidas en programas de política pública orientados a mejorar su bienestar se redujo.

Para concluir se debe indicar que los elementos que clarifican el debate generado a raíz de este texto es mostrarle al lector los elementos que están detrás de la publicación de las cifras de pobreza en el país. Aun así el debate sigue abierto dado que los pobres siguen esperando, en medio de la incertidumbre, que el país los reconozca, no solo en los libros oficiales, sino a través de la generación de oportunidades reales que hagan posible la transformación de sus vidas, no solo por medio del asistencialismo, sino a través de la inclusión en esos espacios vitales como la educación, el empleo y la vida misma. En síntesis, no es suficiente la medición de la pobreza, ya que el verdadero problema sigue persistiendo 
aunque las cifras estén dando la percepción de que su eliminación se aproxima muy pronto.

\section{REFERENCIAS}

Altimir, O. (1979). La dimensión de la pobreza en América Latina, serie Cuadernos de la CEPAL, N²7, Santiago de Chile: Publicación de las Naciones Unidas.

Canberra group (2001). Final Report and Recommendations. Ottawa: Second Editon

Casas, J. \& Barichello, R. (2015). Hacia una noción sobre la pobreza. Apuntes del CENES, 34(59), 39-62.

Cepal (1990). Magnitud de la pobre$z a$ en América latina en los años ochenta. Estudios e informes de la CEPAL, No. 81. Recuperado de: http: //repositorio.cepal.org/bitstream/ handle/11362/33451/S9000548_ es.pdf?sequence $=1$

Cepal (2010). Panorama social de América Latina. Recuperado de: http:// repositorio.cepal.org/bitstream/ handle/11362/1236/S2011800_ es.pdf?sequence $=4$

Coudouel, A., Hentschel, J. \& Wodon, Q. (2002). Medición y análisis de la pobreza. Recuperado de: http://siteresources.worldbank.org/INTPRS1/ Resources/383606-1205334112622/ 4768783-1205337105916/11025_ data_sp.pdf

FAO/OMS/UNU (1985). Necesidades de energía y proteínas. Recuperado de: http://whqlibdoc.who.int/trs/WHO_ TRS_724_(part1)_spa.pdf

Fedesarrollo, (2011). Tendencia Económica. Informe Mensual (112). Recuperado de: http://www.repository.fedesarrollo.org.co/bitstream/11445/608/1/ TE_No_112_Agosto_2011.pdf
Grupo de Río (2007). Grupo de Expertos en Estadisticas de Pobreza. Compendio de mejores prácticas en la medición de la pobreza. Santiago de Chile, Recuperado de: http://www.eclac.cl/deype/ publicaciones/sinsigla/xml/9/34409/ rio_group_compendium_es.pdf

Misión de Expertos para el Empalme de las Series de Empleo y Pobreza - MESEP (2011). Pobreza monetaria en Colombia. Resultados $2^{a}$ Fase. Recuperado de: http://www.dnp.gov.co

Muñoz, M. (1991). La pobreza en trece ciudades colombianas. Recuperado de: http://www.eclac.cl/deype/mecovi/docs/ TALLER13/10.pdf.

Muñoz, M. \& Rivas, G. (2006). Construcción de las canastas normativas de alimentos para trece ciudades, resto urbano y zona rural. Recuperado de: https://books.google.com.co/books/ about/Construcci\%C3\%B3n_de_ las_canastas_normativas.html?id= HdHTtgAACAAJ\&hl=es-419

Muñoz, M. (2011). Pobreza: la confusión es de mucha gente. Recuperado de: http:// www.razonpublica.com/index.php/econom-y-sociedad-temas-29/2435-pobreza-la-confusion-es-de-mucha-gente-. html.

Ravallion, M. (1998). Poverty Lines in Theory and Practice. Recuperado de: https://books.google.com.co/books?hl=es $\& l r=\& i d=V d f W 0 H z e q n 0 C \& o i=$ fnd \&pg= PR7\&dq=Poverty + Lines + in + Theory + a nd+Practice \&ots $=$ ZUFCy $4 \mathrm{ftVb} \&$ sig $=\mathrm{d} 2$ $\mathrm{dPnCNVb-Li61aL2TMojudGTaQ \# v=0}$ nepage\&q=Poverty\%20Lines\%20in\%20 Theory $\% 20$ and $\% 20$ Practice $\& \mathrm{f}=$ false

Rowntree, B. (1901). Poverty: A Study of Town Life. London: Mcmillan.

Sen, A. (1992). Sobre conceptos y medidas de pobreza. En el conocimiento de la pobreza en América Latina. Revista Comercio Exterior, 42(4), 310-322. 


\title{
METHODOLOGIES FOR THE ESTIMATION OF ABSOLUTE POVERTY LINE IN COLOMBIA: A REFLECTION'
}

\author{
Julian Augusto Casas Herrera ${ }^{2}$
}

Universidad Pedagógica y Tecnológica de Colombia, UPTC - Artícle Tipe 2.

Reflection - Received: June 16th 2015 - Accepted: June 21th 2015

doi: http://dx.doi.org/10.17981/econcuc.36.1.2015.28

\begin{abstract}
This article emerged as a response to the controversial figures published on monetary poverty in Colombia in 2011, according to which three millions of poor people disappeared. We inquired into the root behind the figures: the methodology for calculating the absolute poverty line. Exploring on the methodological elements, found that the methodology employed by the Economic Commission for Latin America and the Caribbean (ECLAC) became in regards to the estimate of poverty in the countries of Latin America. Therefore, this document explains the main differences between the four official methodologies implemented in Colombia on the measurement of poverty. The text shows that the poverty figures serve as targeting instrument for those who are identified as poor; however, it is evident that it can cause methodological changes in the correct identification of the number of poor people. This made the latest methodology implemented in the country, which allows us to conclude that the responsible to carry out the measurement must assume an ethical commitment to give coherence to their estimates with what is perceived in society.
\end{abstract}

\section{Keywords:}

Methodology, Homelessness, Poverty, Colombia.

JEL: B49, D13, D63

\section{If you need to reference this article}

Casas, J. (2015). Methodologies for estimating the absolute poverty line in Colombia: a reflection Economical CUC, 36(1), 217-244. doi: http://dx.doi.org/10.17981/econcuc.36.1.2015.28

\footnotetext{
${ }^{1}$ Article of reflection product of the research entitled «alternative proposal for lines of destitution and poverty that exist in Colombia (2002-2011) «, under the line of research economic inequality and social policy, submitted to the National University of Colombia.

${ }^{2}$ Economist and specialist in finance from the Universidad Pedagógica y Tecnológica de Colombia (UPTC).Masterıs Degree in Economics from Universidad Nacional de Colombia. Teacher of the school of economics, UPTC. Email: julian.casas01@uptc.edu.co.
} 


\section{INTRODUCTION}

The motivation for this study arose when La Misión de Expertos para el Empalme de las Series de Empleo y Pobreza (MESEP) (The Mission of Experts for the Connection of the series of Employment and Poverty) and the Departamento Nacional de Planeaciòn (DNP) (National Planning Department) published in 2011 the figures of poverty. These are based on the calculation of the Indigence Lines (IL) and poverty Lines (PL). The first calculates a basket of food, per person and month, whose value was $\$ 78,000$. The second is determined by multiplying the value of the IL by the coefficient of Orshansky (CO), which had a value of 2.4. However, the results generated discontent causing a national debate that arose in connection with the use of the value of the $\mathrm{CO}$, since the MESEP used CO the average of countries in Latin America (2.4), instead of the Colombian CO (2.87), which would make the PL go from $\$ 187,200$ to $\$ 226,200$ and, in consequence, there would be around 19 instead of 16 million poor people. The disappearance of three million of poor justifies the development of this work, such as clarifying element of academic and social debate generated in the country (Muñoz, 2011).

To carry out this clarifying exercise this document aims to explain the main differences between the four official methodologies implemented in Colombia on the measurement of the monetary poverty, since these were derived policies leading to reduce levels of destitution and poverty in the country. The importance of this lies in the fact that small methodological changes produce changes in the correct identification of the number of poor people. The document has four parts, counting the introduction as the first. The second part you will find show the main elements of the methodology of the Economic Commission for Latin America and the Carib- bean (ECLAC) that originated in Latin America (LA) the estimate of poverty. In the third presents the differences between the four methodologies implemented in Colombia. Finally, conclusions are made to reflect the main elements of the document.

\section{ECLAC Methodology}

The methodology of the ECLAC was transformed into a benchmark for the countries of $\mathrm{AL}$ at the time to assess quantitatively the poverty (Altimir, 1979). The approach taken by the ECLAC (1991), to estimate the magnitude of poverty in LA, corresponds to the biological approach ${ }^{3}$ that considers requirements of survival to define a PL as well as a IL, because as said by Sen (1992), hunger is the most obvious aspect of poverty. The estimates are based on the income method or cost of basic needs that compares the income of households regarding the cost of meeting their basic needs.

The estimate of the monetary poverty requires information on income or expenses -lease income or consumption ${ }^{4}$-, which usually comes from the Income and Expenditure Surveys (IES) that perform the countries every 10 or 12 years. The responsible for carrying out the measurement exercise must evaluate the consistency of the in-

\footnotetext{
${ }^{3}$ This came in the wake of the work of Rowntree (1901).

${ }^{4}$ Coudouel, Hentschel and Wodon (2002) point out that if the information of consumption of the income and expenditure surveys (EIG) is detailed, this would be better than the income to measure poverty, for three reasons: (i) consumption is more closely linked to the welfare of the individual; (ii) can be measure better than income; and (iii) better reflects the real standard of living of households, because it reveals the ability to cover their basic needs. However, the use of income allows you to differentiate between the sources of income doing easy comparison with data from other sources, which would be able to verify the quality of the data of the IES. The ideal is to calculate the PL and IL with both to assess the consistency of the results.
} 
come and expenses ${ }^{5}$ since the information comes from a survey, i.e. a sample of the population of the country, which if it involves a margin of error that affects the quality of the estimates.

The approach of the PL has been the most widely used to make quantitative assessments on poverty in the world. The approach to a home classified as poor if its income or expense is less than the value of a PL given. The PL represents the aggregate value of all goods and services deemed essential to satisfying the basic needs of the household. There are three approaches to the PL: absolute, relative and subjective (Homes \& Barichello, 2015). The first is discussed in this document. The estimate of the PL consists of two lines: Food PL (FPL) and Non-Food PL (NFPL).

The calculation of the FLP you select foods that will shape a Standard Food Basket (SFB) that must meet nutritional requirements; while for NFPL there is no objective criterion on a reference value at least. To select the contents of the basket there are two options: normative and seminorm. The first is that the people eat healthfully. The second respects the consumption habits, even if they are not healthy. Given that the latter is the most used describes the three main steps to its construction.

The first step is to determine the rate of basal metabolism, i.e. the requirement or minimum caloric energy ${ }^{6}$ needed to keep an individual alive at rest during a day. This rate is inferred from the information of the socio-demographic structure of the urban and rural population of each country, and specifically finds minimum requirements for calories, protein and nutrients for a person of average age and sex at

\footnotetext{
${ }^{5}$ Is evaluated by observing the degree of underestimation of each one. Opting for the less underestimated.

${ }^{6}$ It is usually measured in kcal daily required by an individual.
}

the national level and by day ${ }^{7}$. Once calculates the weighted average of the calorie needs per person, is passed to determine the total requirements for a home. The requirements arising from recommendations from agencies such as the Food and Agriculture Organization (FAO), World Health Organization (WHO), and the United Nations University (UNU). ${ }^{8}$

The second step makes a selection of a population group objective, better known as the Reference Population (RP) ${ }^{9}$, with the aim to define the composition of the $\mathrm{SFB}$ and calculate Engel coefficient (EC) or the inverse of the $\mathrm{EC}$, i.e. the $\mathrm{CO}^{10}$. To choose this population is organized by the homes, from lowest to highest, according to the value spent on goods. In addition, the selection of the same must meet two conditions:

i. Spending on food must meet the nutritional requirements, respecting the food habits, and slightly exceed the minimum nutritional requirements means estimated; and

ii. Must be capable of assuming other expenses required to meet the most basic needs no food (health, clothing, education, housing, transport).

\footnotetext{
${ }^{7}$ The requirements underestimate the needs of energy and proteins of the households in which the age of its members is higher than the national average, and vice versa.

${ }^{8}$ The recommendations of the FAO and WHO consider the census data of the socio-demographic structure of urban and rural population of the country. The first recommendations on energy needs came from the FAO in 1950. From then on has updated the protein and energy requirements for the years 1958, 1966, 1971, 1981, 1985 and 2001.

${ }^{9}$ The criterion of the ECLAC requires that the households included in it, on the one hand, meet their food needs, and on the other hand, that the habits of consumption in other goods and services are not subject to decisions of a framework of extreme scarcity or abundance economic.

${ }^{10}$ The EC exits to divide the food expense among the total expenditure. While the $\mathrm{CO}$ is obtained by dividing the total cost between the cost of food. The estimate of the $\mathrm{EC}$ or $\mathrm{CO}$ exits the PR.
} 
In the third step provides the content and cost of the food basket. The cost can be determined in two ways: (i) to design a basket and give it a price, ii) calculate the price of the basket without specifying their content. The first mode, the most common, determines the food that will compose the SFB analyzing for each food three aspects based on household consumption patterns: (i) acquisition frequency for along with the percentage of households which was purchased; (ii) monthly cost per person in that well, that is the percentage of the expenditure that represents in the total spending on food; and (iii) physical quantities purchased expressed by equivalence gramsday per person.

Of the foregoing, arising from the criteria for inclusion of foods ${ }^{11}$ that are used to determine the presence or absence of each food item within the SFB. Basically, what is done is to take each of the foods registered in the IES and checked if the food, at least satisfies one of the criteria and if so, the food passes into the basket. Once inside the basket determines the food group $^{12}$ that belongs to the food.

ECLAC (1990) assured that the food of the SFB had at least $10 \%$ of calories and protein between $15 \%$ and $25 \%$ fat calories; that the cereals and pulses do not contribute more than $60 \%$ of the total calories;

\footnotetext{
${ }^{11}$ ECLAC (1991) works with five inclusion criteria, but each country is autonomous to take as many criteria as you consider to be relevant. The criteria are: (i) if the food is consumed by $30 \%$ or more of the homes; (ii) if the food represents at least $1 \%$ of the total expenditure on food; (iii) if the food contributes at least $1 \%$ of the total calories or protein consumed; (iv) if the food represents $0.5 \%$ or more of the total weight of the food purchased by households; and (v) if the cost of the food represents, at least $5 \%$ or more within the group to which it belongs the food.

12 ECLAC (1991) classifies 10 food groups: flours and cereals; dairy products; meat, poultry and eggs; fruits; vegetables; legumes; fat; tubers, roots and bananas; sugar; and others. In the latter meet those foods with lower power consumption.
}

and to ensure that the quality of protein is adequate, at least $35 \%$ of the same, they would have to be of animal origin. Equally, the SFB from each country were evaluated in terms of the content of seven nutrients: calcium, iron, retinol (vitamin A lease), thiamin (vitamin B1), riboflavin (vitamin B2), niacin (vitamin B3), and ascorbic acid (vitamin C).

Immediately it is necessary to check if the content of the SFB exceeds caloric requirements identified by the calorie rule, supplied by the FAO, and if so is passed to make adjustments caloric, protein and dietary to each one of the foods that make up the SFB. In this way, sets forth the amounts of calories ${ }^{13}$ and nutrients needed for each food; obtaining the SFB of each country ${ }^{14}$. It is important to indicate that the SFB does not represent an ideal diet, but you are looking for: (i) reflect the consumption patterns of households of the PR, (ii) prevent diseases associated with inadequate nutrition, and (iii) obtain a SFB at a lower cost. However, it is necessary to clarify that if they have to make adjustments to the SFB, these must favor the habits of the population rather than the nutritional standard and the cost.

The valuation of the SFB is done using the implicit price of the own IES RP, which depart from split the cost of the food between the quantity purchased of the same. It should explain two aspects. On the one hand, if the goal is to get a basket cheaper expensive foods are replaced by other

\footnotetext{
${ }^{13}$ Suppose that the FAO establishes that calories must be consumed per day are from 2200 , but the content of the CNA sheds 2300 . Then it becomes split 2200 between 2300 , which gives an adjustment factor of 0.95 that you will have to multiply to each food item to the CNA is snug to the standard caloric (2200). Adjustments are made with the order that is not problems of overweight, undernourished, among others.

${ }^{14}$ According to the information existing in the country are obtained both CNA and EC for the urban and rural domains.
} 
cheaper of the same food group. On the other hand, the FPL usually represents the cost per person is required to comply with the caloric requirement of the average population. Here it should be noted that in the approach of the LP the unit of analysis is the household, but not the individual. This is a limitation because it is impossible to assess the poverty at the individual level.

The alternative to get around this limitation passes by associating the LP of the home through the LP per capita. However, this assumes that the monetary cost to meet the needs of an individual is homogeneous for all members of the household and that there are no economies of scale in consumption. In addition, the approach of the LP requires equivalence scales to add the results at the national level. The equivalence scale more used is the $\mathrm{EC}^{15}$, which is essential for estimating the LPNA. In fact, to build the LP and LI is requires that the information of the EIG meets Engels law ${ }^{16}$, and that estimates of the different items of expenditure are within reasonable levels when compared with other sources of information ${ }^{17}$ (similar surveys). Before concluding this section should specify two more things: non-food requirements, together with the correction and adjustment of the income.

As already said, there is no objective criterion to determine a reference value for the minimum LPNA or non-food requirements. However, the most com-

\footnotetext{
15 This assumes that the division between the cost of feeding and the total expenses of two households is the same. Well this index expresses that the cost of maintenance of the first home in comparison with the second is the same. See Rio Group (2007).

${ }^{16}$ Indicates that as income increases, the costs that are dedicated to the most basic necessities are declining, while those who are devoted to luxury goods are increasing.

${ }^{17}$ This is what is called external consistency of the EIG. This point was the controversial in the latest methodology implemented in Colombia. See Methodology 2011 later.
}

monly used method for the establishment LPNA is based on the EC observed of the PR. This is based on the work of Mollie Orshansky who established the LP of the United States; therefore it is sometimes called $\mathrm{CO}^{18}$. Note that the LP covers food, through the LPA, and in addition is aware that the homes are more than food. This consciousness takes shape with the value of the EC that intended to cover the items of housing, transportation and clothing ${ }^{19}$. So if you divide the value of the LPA between the EC will be the final value of the LP; or it can also be obtained if you multiply the LPA by the $\mathrm{CO}^{20}$.

On the other hand, certain the LI and LP and given the problems that have the EIG $^{21}$ ECLAC decided to assess the magnitude of the biases inherent in the amounts of income declared to adequately use the income data and, in consequence, step to make a correction and adjust-

\footnotetext{
18 This has several implications. On the one hand, according to the INE (undated) implies "that the homes that they can adequately cover your power needs met, at the same time, the minimum standards for other basic needs, something that is not necessarily supported by empirical evidence (...) [On the other hand, indicates that] there may be an inconsistency in this way to get to a poverty line. The minimum requirements are derived from power in legislative form, calculating how much it would cost the minimum requirements; while the non-food items are determined by looking at reality as it is spending the people. To eliminate the inconsistency, we would have to assume that what the people really comes to spend is what you need to spend in the non-food item, a course clearly unrealistic".

${ }^{19}$ Within the non-food basket it is included a portion of the income for the wardrobe that searches for harboring a wardrobe essential; it also includes a portion for housing of two and three bedrooms, which is sheltering a part for the lease of a reference family, along with the payment of public services and amenities (fridge, stove, washing machine); and includes a component for the transport of the members of the family of reference to go to work, to school, shopping, among others (INE, undated).

${ }^{20}$ Currently, ECLAC urban $\mathrm{CO}$ works with a value of 2 , and rural 1.75 .

${ }^{21}$ Such as the quality and accuracy of the measurement of income, geographic coverage, representativeness of the sample, the lack of response to questions of income, the biases and errors associated with the sampling.
} 
ment of income. For this he went to the Adjustment to National Accounts (ANA) that compares the items of income of the EIG with those resulting from an estimate of the income and expenditure account of the homes of the system of national accounts; as well only if the first are above the last generated coefficients of adjustment in accordance with the discrepancy for each source of income, and thus easing the biases by sub-statement or by the lack of response to questions about the income of employees, independent workers and retirees.

In summary, the first and third step serve to define the monetary value of the $\mathrm{LI}$, while the second step is to give the $\mathrm{CO}$ by which is multiplied to give the LP. So that people who do not have an income equal to or greater than the value of the LI, shall be deemed indigent or extremely poor. In contrast, those who are above the LI and below the value of the LP is considered poor.

Finally, it should be pointed out three things. The first is that the EIG are carried out every 10 to 12 years, given that its cost is very high. Therefore, the values of the LI and LP were updated, in the years that there is no survey, in accordance with the cumulative change in the Consumer's Price Index (CPI) for each country. ECLAC (2010) points out that until December 2006 he applied the same variation to both lines, but since 2007 the LI is updated with the change in the CPI for food, and the LP with the CPI for each year. The second is that since 2005 the ECLAC (2010) began to revise the methodology that has been used since the eighties, for four reasons:

i. The LI and LP are derived from patterns of consumption of the EIG of those years. Therefore, you must build new CNA that adhere to the habits of the EIG more recent; ii. It is required to introduce methodological changes that reflect advances in the measurement of poverty in the world (harvesting techniques, among others);

iii.It is necessary to extend the concept of income of the EIG used; and ${ }^{22}$

iv. Make the revision of the ACN, already is not currently considered essential that you do this.

And the third is due to the aims in the estimate of the LP and LI, which are looking for: i) quantify the magnitude of poverty, (ii) identify the homes in such a situation, and (iii) provide information for the design of public policies aimed at reducing both want and poverty. It means that the information that shows the classification of the number of poor and indigent serves as an instrument of targeting to governments, through programs of public policies, alleviating the negative effects that generate poverty. ${ }^{23}$

\footnotetext{
${ }^{22}$ This concept has been discussed in the report of the Canberra Group (2001).

23 In Colombia A major groups can be classified: (i) Conditional Cash Transfers, among these are: Families in Action and Social Protection Program to older adults; (ii) Care and Child Development: Comprehensive Care Program for the Early Childhood Program and Community Homes; (iii) food and Infant Nutrition: School Feeding Program, Children's breakfast program with Love; (iv) Education: Program credit access and program services free of charge; v) health: health subsidy scheme; (vi) Employment: Youth Action Program, Program and Rural Youth Unemployment Subsidy Program; (vii) Economic and Social Inclusion: Rural program opportunities, Comprehensive Program Grant of Lands, Housing Program of Social Interest, Rural Housing Program, Network for Overcoming Extreme Poverty, (viii) Elderly: National School Feeding Program for the Elder "Juan Luis Londoño de la Cuesta"; (ix) Poor: Food Security Network (see: http://estadisticas.comunidadandina.org/ estadisticasandinas/CANPS/default.aspx). In addition, the Conpes associated with poverty are numbers $102,102,111,135,150$ and 217. The reader can visit the following link to see the Conpes aproved: https:// sisconpes.dnp.gov.co/DocumentosConpesAprobados/ IraDocumentosConpesAprobados/tabid/166/Default. aspx
} 


\section{METHODOLOGIES EMPLOYED IN COLOMBIA}

This section explains the aspects that were taken into account in the four methodologies implemented in Colombia for the approach of the LP absolute, for 1988, 1998, 2005 and 2011. The document takes as regards the work of Munoz \& Rivas (2006) and of the MESEP (2011). The first job serves as a benchmark for the methodologies of 1988 (M88), 1998 (M98) and 2005 (M05). The second describes the methodology of 2011 (M11).

The work of Munoz \& Rivas (2006), shows a comparison, based on their values and characteristics, obtained between the baskets with the M88 and M98. The need arose because comparative for 1998 was estimated the LP and LI with the EIG 1994-1995 (EIG94- 95). In addition, this estimation is conducted using a different methodology than the one used in 1988 to work with the EIG 1984-1985 (EIG8485); the foregoing originated as a result that the CNA 1998 were more expensive than the 1988. The 1998 estimates of the LI and LP were not published; but the National Bureau of Statistics (DANE) updated annually their values, and find that by 2004 a $10 \%$ more than poor with the M98, in comparison with the M88.

The dispute had an important eco and it was decided to investigate the differences between the M88 and M98, giving as a result the M05. This comparison built a new CNA, but applying the M88 with the data of the EIG94-95. To make understandable the differences between the four methodologies identified is passed to describe their main aspects, highlighting that the differences lie in four aspects: (i) PR; (ii) criterion for the inclusion of foods to the CNA; (iii) determination of the necessary quantities of the foods that make up the CNA; and (iv) calculation of the LP.

\section{Methodology 1988}

The main criticism that generated the M88 had to do with the election of the $\mathrm{PR}$, since the construction of the CNA chose the consumption habits of the poorest households and, therefore, the method is based on a selection of the poor, which methodologically requires that the households included should not be confronted with a framework of extreme scarcity or abundance of economic. In fact, the M88 took as $P R$ between the percentiles 1 to 25. Before moving on to point out the criterion for the inclusion of foods to the CNA, Muñoz (1991), reveals that a CNA must have four characteristics: (i) to meet the minimum nutritional requirements; (ii) respect, to the extent possible, the eating habits of the PR; (iii) take into account, as far as possible, the availability of food; and (iv) have a minimum cost. In this regard, the M88 took into account four criteria for the inclusion of foods. As well, a food came in the CNA if at least met one of the following criteria:

i. It consumed $30 \%$ or more of the homes,

ii. Represented at least $1 \%$ of the total food expenditures,

iii.Provided at least $1 \%$ of the total calories or protein consumed, and

iv. Represented 0.5 per cent or more of the total weight of the food purchased by households.

For the determination of the necessary amounts of the food that the CNA is mega took into account the nutritional requirements of the Colombian population, which is governed by one side, per the guidelines of the FAO/WHO/UNU (1985), with information provided by the Colombian Family Welfare Institute, specifically on height and weight. In addition, the calories and protein M88 took into account seven nu- 
trients: calcium, iron, retinol, thiamine, riboflavin, niacin, vitamin $\mathrm{C}^{24}$.

It is important to indicate that the M88 decided to work with information of current expenditure for home, rather than income, because according to Muñoz (1991) "the cost information is more reliable than the income, since the latter presents a greater degree of underestimation. This fact is reflected in that the proportion of poor people is less, for twelve of the thirteen cities, when you take the expenditure as a reference, (...) exception of Medellin”.

With the inclusion criteria and certain amounts of food, valued the CNA or LI. In addition, introduced three nutritional restrictions in order that there was a balance between calories and protein, which was established in the following way: (i) the proteins of animal origin should be between $20 \%$ and $45 \%$, (ii) the calories from fat should be between $20 \%$ and $30 \%$, protein between $8 \%$ and $12 \%$, and carbohydrate between $55 \%$ and $65 \%$; and (iii) the sugar and the panela should not contribute more than $20 \%$ of the energy obtained from all sources of carbohydrates (Muñoz, 1991).

With theses restrictions the food that would comprise the CNA were classified in nine groups: dairy (milk and cheese); meat and eggs; legumes; cereals; tubers, roots and bananas; vegetables; fruits; fats; and sugars. Finally, with respect to the calculation of the LP was estimated by dividing the LI between the EC. With these criteria were estimated the LI and LP for thirteen cities, which were updated annually with the CPI.

\footnotetext{
${ }^{24}$ The average requirements of calories and nutrients per capita of the Colombian population of calories, protein, and micro-nutrients were: calories (2209), proteins (62 $\mathrm{mg}$ ), calcium (874.6 mg), iron (16.4 mg), niacin (15.1 mg), retinol $(820 \mathrm{mg})$, riboflavin $(1.36 \mathrm{mg})$, thiamine $(1.2 \mathrm{mg})$, vitamin C (56.1 mg).
}

\section{Methodology of 1998}

The CNA were built, with the EIG9495, taking into account the critical that generated the M88. Therefore, the M98 change the $P R$ and added a criterion for the inclusion of foods in the CNA. With regard to the first change, took the consumption habits of the average between 1 to 90 percentile of the population. With regard to the second change, according to Munoz \& Rivas (2006), the low diversity of foods from the M88 led him to introduce a new selection criterion. The new approach included food in the CNA if the cost of this represented, at least $5 \%$ or more within the group to which it belongs the food.

The determination of the necessary amounts of the food that would comprise CNA of M98 change with regard to the M88 since the recommendations average calorie, protein, and micro-nutrients were thus: calories (2297), proteins (62), calcium (620), iron (16), niacin (16.1), retinol (775), riboflavin (1.4), thiamin (1.2), vitamin C (53). Note that in the M98, were reduced the nutritional requirements of calcium in $29 \%$, iron $2.4 \%$, retinol 5.5 $\%$, and vitamin C $5.5 \%$; in contrast, in calories, niacin and riboflavin increased by $4 \%, 6.6 \%$ and $2.9 \%$ respectively. In proteins and thiamine there was no change. Due to these changes the CNA of the M98 were more expensive than those of the M88.

Finally, for the estimate of the LP was changed the calculation of the EC, which was reflected in the following equation of Engel for food: in which represents the participation of food expenditures on total spending (i.e., the EC) and is the total current expenditure of the household. Well if it is replaced in the equation the value of the LI, is the total expenditure and, therefore, w; thus obtained the LP. 


\section{Methodology of 2005}

The M05 returned to take as $P R$ and $25 \%$ of the poorest; the criterion for the inclusion of foods to the CNA was the same one used in the M88; for the determination of the necessary amounts of the food that the $C N A$ is mega took into account the nutritional requirements of the Colombian population, guided by the guidelines of the $\mathrm{FAO} / \mathrm{WHO} / \mathrm{UNU}$ (1985). It is essential to note that the M05 was developed from two sources of information: (i) for the LP urban use the EIG94-95 and (ii) for the LP rural used the information of the first follow-up to the families in action program of 2003. The calculation of the $L P$ was the same way as in the M88.

In addition, the M05 estimated values of LI and LP for 13 cities, the rest (10 cities) and $^{2526}$ for the rural area. In regard to the LP of the rural area, it should be noted that in order to build the LI, Munoz and Rivas (2006) warn:

"We do not really know how it was obtained the rural poverty line used until now; only you have the data of the source of information, that was the Survey of Food and Nutrition 1981, but it is not known the methodology nor any document on it. [Unfortunately], the cost information or consumption of food is too scarce to the rural area, therefore, it is difficult to have information on how to build the baskets regulations".

For this reason, indicate that one of the advantages of the M05 lies in the fact that thanks to the assessment of the impact of the program families in action were made in 2002 and 2003, surveys to the poorest

\footnotetext{
${ }^{25}$ Bogotá, Medellín, Cali, Barranquilla, Bucaramanga, Manizales, Pasto, Cartagena, Cúcuta, Pereira, Montería, Neiva and Villavicencio.

${ }_{26}$ Tunja, Florencia, Popayán, Valledupar, Quibdó, Riohacha, Santa Marta, Armenia, Sincelejo and Ibagué.
}

rural households in strata 1 and 2 of the System of Potencial Beneficiaries for Social Programs (SISBEN). The 2002 was called baseline (LB) and the 2003 first follow (PS). This fact allowed to build the CNA rural, because the information of the survey of PS, according to Munoz \& Rivas (2006), had no information on the acquisition of food on the part of households.

Two further points must be noted on the survey of PS. On the one hand, discussed two types of municipalities: control and treatment. But it is preferred to work with the municipalities treatment because the homes of these had expansion factors. On the other hand, to assess the amount obtained from food purchased ${ }^{2728}$ is not use the implicit price average of these of the municipality to which corresponded the home. In addition, the development of the rural CNA had two phases: (i) took the total of the PR of the municipalities treatment; and (ii) for the estimation of prices and quantities, specifically, with which they are purchased used implicit prices average of each food.

The criterion for the inclusion of foods to the CNA rural handling two forms of selection: the M88 and the methodology ECLAC. The main discrepancy between the two is that the second, the foods that are not included directly in the CNA is included under the heading called other. That is to say, while the M88, M98 and M05 grouped the food of the CNA in nine groups, the methodology ECLAC grouped in ten.

The determination of the necessary amounts of the food that mega the CNA was made in the same manner as the M88. However, there was a modification that

\footnotetext{
${ }^{27}$ An expansion factor is the number of persons in the population, which represents a person in the sample.

${ }^{28}$ That is to say, those who are gifted, auto-consumption or auto supply.
} 
took into account an average based on the structure by age and sex of the rural population. This information came from the population census of 1993. Muñoz \& Rivas (2006) commented that in assessing the CNA with each one of the methodologies that are with the M05 the value of the LI, for the month of September 2003, was $\$ 2113.1$ per capita/day; however, with the ECLAC the value was $\$ 2237.7$.

To make the comparison between the two methodologies is found that the LI of the ECLAC was better fitted in the vast majority of nutrients - less protein-, hence this basket is preferred in spite of the fact that its cost will exceed the CNA of the M05 at $\$ 124.6$ per day. Finally, to move from the LI to the LP the M05 followed exactly to the M88, but keep in mind that the PR for the rural area was the total survey of the PS.

Two final clarifications are necessary: on the one hand, the EC in the M05 for all of the cities was higher compared to the M98, due to the PR that was used. On the other hand, the M88, M98 and M05 used the ACN, which contrasts the amounts of income by source of income (wages, profits, and others) of the EIG, with respect to the amounts of the same sources of national accounts of the country. Thus, the contrast was obtained from adjustment factors applied to the income of the EIG in order to adjust or correct the problem of sub-statement that is recorded in the IGE.

\section{Methodology $2011^{29}$}

According to the designers of the M11 was necessary to modify the LP by six

\footnotetext{
29 This section is based on the document entitled «monetary poverty in Colombia: New methodology and figures 2002-2010», presented by the MESEP (2011).
}

reasons: (i) to deploy sophisticated statistical methods to impute the missing data; (ii) delete the ACN; (iii) include recent methodological advances, to obtain a more precise way to measure poverty in the country; (iv) update the consumption habits, since it had a new EIG, namely the national survey of income and expenditure 2006-2007 (ENIG06- 07); (v) incorporate the recommendations of the recent $\mathrm{FAO}$; and (vi) achieve comparability with other Latin American Countries. $^{30}$

The M11, in contrast to the M05, built the urban and rural LP with the same source of information (ENIG06- 07), which according to the MESEP ensured a better comparability between the rural and urban data. The M11 assessed the internal consistency of the external and ENIG06-07, through the estimation of Engel curves for the first; and for the second, compared the distributions of different categories of expenditure compared to those obtained in other surveys. The members of the MESEP determined that the ENIG06-07 has internal consistency, but not external. In fact, they argue that in the ENIG06-07 there is the underestimation of some categories of expenditure, in comparison with other sources that capture similar information, up to $50 \%$. This conclusion was the determining factor for choosing a CO exogenous to the urban area, and maintains the proportion of $\mathrm{CO}$ rural. In that order of ideas, the

\footnotetext{
${ }^{30}$ Note that you will step to the EIG the ENIG since DANE for 2006-2007, it joined the household survey with the EIG. However, according to Nunez, quoted by Fedesarrollo (2011), expresses that this integration has brought several problems such as the ENIG is made on a sample of $25 \%$ of households in the survey of households, the sample of the household survey is changed significantly and the revenues were unexplained variations along the distribution of income, and the data reported income had to be discarded for 2006 and 2007, which resulted in that there were no poverty estimates for those years.
} 
M11 continued six steps to build the LP and LI: ${ }^{313233}$

i. Determination of the list of current expenditure per capita per unit of expenditure;

ii. Construction of a spatial price deflator, to establish the relationship between urban and rural domains and, when applied, could be a single PR;

iii. Management of all the households by the current expenditure per capita deflated to determine a PR, taking $45 \%$ of the incident, which was located between the percentiles 30 to 59 ;

iv. Construction of the NAC, which were adjusted to the calorie requirements $^{34}$ together with three criteria for the inclusion of foods;

v. Valuation of the CNA or LI to the median of the implied prices; and

vi. Multiplication of the exogenous $\mathrm{CO}$ by the LI for the LP.

The PL and IL were updated monthly by means of annual inflation. It should be noted that with the expansion of the levels of geographical breakdown, implemented by DANE (National Administrative Statics Department) with the integrated household

\footnotetext{
${ }^{31}$ Some of them are: the EIG1994-1995, the quality of life survey 2003 , among others.

32 The members of the MESEP note, for example, the undervaluation of the food category is stated by comparing the average spending on food of the ENIG06-07 with the Quality of Life Survey CVD08 the difference is significant, $\$ 287,000$ for the first and $\$ 654,000$ for the second.

${ }^{33}$ The average urban $\mathrm{CO}$ was 2.4 , although the $\mathrm{CO}$ that threw the ENIG06-07 was 2.87, while the rural CO was 2.09. What was done in the M11 was to keep the proportion and so conditioned CO rural to the value of 1.74 , which came out to make the following rule of three: COrural $=\left(\frac{2.09 * 2.4}{2.87}\right)$.

${ }^{34}$ The caloric content of the basic basket was obtained by multiplying the quantities consumed by the caloric value of each foodstuff, which had as a source the tables of the Colombian Family Welfare Institute 2000, 2005, FAO and DANE.
}

survey from 2006 and due to the availability of information of the CPI, it was unable to go beyond 13 to 24 cities. In other words, the M11 has updated the LP and BO for 26 domains, divided into two groups: 25 urban domains and 1 in the rural area. ${ }^{35}$

The M11 took as $P R$ the percentiles 30 to 59 of the distribution of per capita expenditure. Interative Ravallion for the selection of this method was applied (1998), which means a percentage of the incidence of poverty for the initial national total around which would focus the population, which was defined in the $45 \%$. This process verifies the validity of the $\mathrm{PR}$, from the check of the incidence of poverty. Well, if the new percentage of poor is within the range defined for the PR a priori (percentiles 30 to 59), the process is completed and asserts that the LI and LP. If it falls outside the range the process is repeated by choosing a PR focused on the new percentage of incidence found with the new lines.

For the inclusion of foods to the CNA, the M11 took the ENIG06-07 the consumption habits of the urban and rural domains of the $\mathrm{PR}$ for the purpose of constructing the CNA in each domain. The M11 included foods that comply with at least one of the following three criteria:

i. It consumed $30 \%$ or more of the household;

ii. It represented at least $1 \%$ of the total food expenditures; and

iii.It contributed at least $1 \%$ of the total calories consumed.

For the determination of the necessary amounts of the food that would comprise the CNA the nutritional requirements according to the recommendations of the $\mathrm{FAO} / \mathrm{WHO} /$

\footnotetext{
${ }^{35}$ The 13 traditional areas are: Medellín, Bogotá, Barranquilla, Cartagena, Manizales, Montería, Villavicencio, Pasto, Cúcuta, Pereira, Bucaramanga, Ibagué and Cali. The new cities are: Tunja, Florence, Popayán, Valledupar, Quibdó, Neiva, Riohacha, Santa Marta, Armenia and Sincelejo. Finally, the other domains are other headers, rural and national.
} 
UNU 2001 were taken into account. In the M11 the CNA was adjusted to reach caloric requirement. This is accomplished by using the quantities observed in the PR and comparing it with the standard caloric that advises FAO for each domain. In Colombia, the average caloric requirements were obtained from the structure of the population by age and sex of the ENIG06-07. The norm for the caloric urban domain determined 2090 calories per day, and for the rural stood in 2049. To comply with the rule had been applied an adjustment factor of 1,969 to the quantities of the urban domain and 0.9242 for the rural domain. In addition, note that the CNA of the M11 did not take into account the seven micronutrients or other requirements of proteins. This is to say they were only adjusted for calories.

The LP was obtained from the multiplication between the LI and CO; however, it should be made clear that the $\mathrm{CO}$ can be endogenous estimate or exogenously. If you go to the first form, this would come out of the information of the EIG; if you go to the second way, is used secondary information. The M11 has opted for the latter option, taking data calculated by the ECLAC for the countries of AL, because in the opinion of the MESEP the ENIG06-07 presented problems of external consistency, as evidenced in the underestimation of the total expenditure on food, which is crucial for the estimation of the CO.

The members of the MESEP decided to use a CO exogenous $2.4^{36}$ at the urban level, and 1.74 at the rural level. Well, the LP urban for 2007 was obtained by multiplying the LI $(\$ 73,984)$ and the CO (2.4), which resulted

\footnotetext{
${ }^{36}$ The countries that were used to make the average of the $\mathrm{CO}$ for the urban area were: Argentina (2.6), Bolivia (2.2), Brazil (3.5 ), Chile (3), Costa Rica (2.8), Ecuador (2.2), Guatemala (2.5), Honduras (2.2), Mexico (2.8), Nicaragua (1.9), Panama (2.8), Paraguay (2.4), Peru (2.2), Dominican Republic (2.3), Uruguay (3.4). To make this average does not 2.4 , but 2.59 . However, when applying the criteria of the ECLAC called pattern purified, it is removed the data from Brazil, resulting in 2.35 , but was adjusted upward, leaving 2.4 .
}

in $\$ 177,562$; and the LP of the rural product between the LI $(\$ 60,968)$ and the CO $(1.74)$, giving $\$ 106,084$. These values are pesos of March 2007. Therefore, the values of the LP and LI were calculated for the previous years (2002-2005) and later (2008-onwards) to the ENIG06-07. Finally, to update the monthly values of the $\mathrm{LI}$ is the M11 using the CPI for food, while that for the LP used the CPI of low income.

Finally, it should be noted that the exercises are derived from methodological exposed in daily values of the CNA (which is composed of several foods) or LI along with a value of the EC or the CO. The results obtained by each of the methodologies implemented in the country are summarized in table 1 .

The above analysis concludes that the estimates of the LI and LP are susceptible to variations depending on methodology. In fact, the debate in the country was generated as a result of some methodological elements of the M11. Perhaps the most questionable was the use of an exogenous CO (2.4), because, as said Núñez, quoted by Fedesarrollo (2011): do not take the [] CO that comes from the survey, in some way, is to accept that the data are not reliable as a whole. Therefore, when not using the endogenous CO (2.87) the poverty for the different domains of the country were estimated and, therefore, the number of people that should be included in programs of public policy aimed at improving their welfare is reduced. Then, note that methodological changes impacted on the correct identification of the number of poor people in the country. This change of $\mathrm{CO}$ caused 3 millions of poor disappear by 2010 .

Likewise, it is remarkable because the MESEP did not take into account the $\mathrm{CO}$ of Brazil (3.5), because, according to the MESEP was very high. If it had been taken into account they would be talking about an urban $\mathrm{CO}$ of 2.59, which would have meant higher poverty rates. So while the figures show that poverty in the country 
Table 1.

LI and LP per capita, by day and month, of the M88, M98, M05 and M11

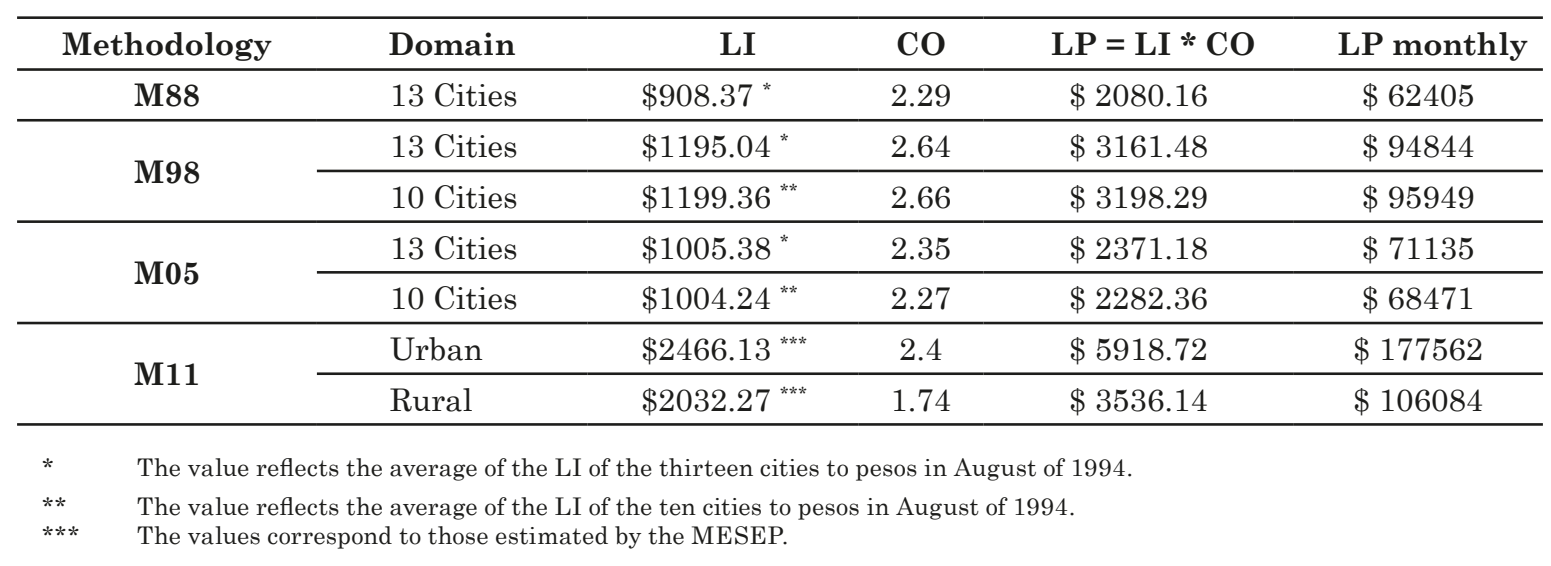

Source: own construction with data of Munoz \& Rivas (2006), Muñoz (1991) and MESEP (2011)

has been reducing the perception of reality says otherwise. Therefore, those responsible for carrying out the measurement of poverty must assume an ethical commitment to give coherence to their estimates with what is perceived in society, because what is at stake is the humanity and dignity of the most vulnerable people in a society already that the official data of poverty served as an instrument of public policy to lessen the negative effects it generates in the existence of the poor, through grants to those who are identified as poor or indigent.

\section{CONCLUSIONS}

This document had motivation as the debate generated by the published figures on poverty in 2011 obtained by the MESEP. That debate is magnificent due to the alleged disappearance of around three million poor. This justified investigate because of their disappearance, found that the approach used by Colombia, to make quantitative assessments of poverty, is the LP absolute. That approach was based on the methodology of the ECLAC. They were also the main methodological elements that took into account the four exercises that have progressed measurements of poverty in Colombia, showed differences between the same.
In this way, it is evident that the estimates are subject to methodological variations. Particularly there was at least an element of methodology of M11 that is questionable (the use of exogenous CO), which had an impact on the results presented by the MESEP and, therefore, in the economic policies of them were derived. So when not using the endogenous CO levels of poverty were lower for the different domains of the country and, therefore, the number of people that had to be included in programs of public policy aimed at improving their welfare is reduced.

In conclusion it should be noted that the elements which clarify the debate generated as a result of this text is to show the reader the elements that are behind the publication of the figures of poverty in the country. Even so the debate is still open because the poor are still waiting, in the midst of uncertainty, that the country recognize these, not only in the official books, but through the generation of real opportunities that make possible the transformation of their lives, not only by means of handouts, but through the inclusion in these vital areas such as education, employment and life itself. In summary, it is not enough the measurement of poverty, because the real problem is still present although the figures are giving the perception that its elimination is approaching very soon. 


\section{REFERENCES}

Altimir, O. (1979). La dimensión de la pobreza en América Latina, serie Cuadernos de la CEPAL, $N^{\circ}$ 27, Santiago de Chile: Publicación de las Naciones Unidas.

Canberra group (2001). Final Report and Recommendations. Ottawa: Second Editon

Casas, J. \& Barichello, R. (2015). Hacia una noción sobre la pobreza. Apuntes del CENES, 34(59), 39-62.

Cepal (1990). Magnitud de la pobreza en América latina en los años ochenta. Estudios e informes de la CEPAL, No. 81. Retrieved from: http:// repositorio.cepal.org/bitstream/ handle/11362/33451/S9000548_ es.pdf? sequence $=1$

Cepal (2010). Panorama social de América Latina. Retrieved from: http:// repositorio.cepal.org/bitstream/ handle/11362/1236/S2011800_es. pdf?sequence $=4$

Coudouel, A., Hentschel, J. \& Wodon, Q. (2002). Medición y análisis de la pobreza. Retrieved from: http:// siteresources.worldbank.org/ INTPRS $1 /$ Resources/383606$1205334112622 / 4768783$ 1205337105916/11025_data_sp.pdf

FAO/OMS/UNU (1985). Necesidades de energía y proteínas. Retrieved from: http://whqlibdoc.who.int/trs/WHO_ TRS_724_(part1)_spa.pdf

Grupo de Río (2007). Grupo de Expertos en Estadísticas de Pobreza. Compendio de mejores prácticas en la medición de la pobreza. Santiago de Chile, Recuperado de: http://www.eclac.cl/deype/ publicaciones/sinsigla/xml/9/34409/ rio_group_compendium_es.pdf
Misión de Expertos para el Empalme de las Series de Empleo y Pobreza MESEP (2011). Pobreza monetaria en Colombia. Resultados $2^{a}$ Fase. Retrieved from: http://www.dnp.gov. co

Muñoz, M. (1991). La pobreza en trece ciudades colombianas. Retrieved from: http://www.eclac.cl/deype/mecovi/docs/TALLER13/10.pdf.

Muñoz, M. \& Rivas, G. (2006). Construcción de las canastas normativas de alimentos para trece ciudades, resto urbano y zona rural.

Muñoz, M. (2011). Pobreza: la confusión es de mucha gente. Retrieved from: http://www.razonpublica.com/index. $\mathrm{ph}$ /econom-y-sociedad-temas29/2435-pobreza-la-confusion-es-demucha-gente-.html.

Fedesarrollo (2011). Tendencia Económica. Informe Mensual (112). Retrieved from: http://www.repository.fedesarrollo.org.co/bitstream/11445/608/1/ TE_No_112_Agosto_2011.pdf

Ravallion, M. (1998).Poverty Lines in Theory and Practice. Retrieved from: https://books.google.com.co/books?h $\mathrm{l}=\mathrm{es} \& \mathrm{lr}=\& \mathrm{id}=\mathrm{VdfW0Hzeqn0C \& oi=}$ fnd\&pg $=$ PR7\&dq=Poverty + Lines $+\mathrm{i}$ $\mathrm{n}+$ Theory+and+Practice\& ot $\mathrm{s}=\mathrm{ZUF}$ Cy4ftVb\&sig $=\mathrm{d} 2 \mathrm{dPnCNVb}-\mathrm{Li} 61 \mathrm{aL}$ 2TMojudGTaQ\#v=onepage\& $\mathrm{q}=$ Poverty\%20Lines\%20in\%20Theory\%20 and $\% 20$ Practice $\& f=$ false

Rowntree, B. (1901). Poverty: A Study of Town Life. London: Mcmillan.

Sen, A. (1992). Sobre conceptos y medidas de pobreza. En el conocimiento de la pobreza en América Latina. Revista Comercio Exterior, 42(4), Ciudad de México: Banco Nacional de Comercio Exterior. 